\title{
Hepatobiliary Iminodiacetic Acid Scan
}

National Cancer Institute

\section{Source}

National Cancer Institute. Hepatobiliary Iminodiacetic Acid Scan. NCI Thesaurus. Code C152066.

Radionuclide imaging of the liver, gallbladder, and bile ducts using the radioactive tracer 99mT c-hepatic iminodiacetic acid. 